\title{
Can resistance against quorum-sensing interference be selected?
}

\author{
Rodolfo García-Contreras ${ }^{1}$, Toshinari Maeda ${ }^{2}$ and Thomas K Wood ${ }^{3}$ \\ ${ }^{1}$ Department of Microbiology and Parasitology, Faculty of Medicine, Universidad Nacional Autónoma de \\ Mexico, Mexico City, Mexico; ${ }^{2}$ Department of Biological Functions Engineering, Kyushu Institute of \\ Technology, Kitakyushu, Japan and ${ }^{3}$ Department of Biochemistry and Molecular Biology, Pennsylvania \\ State University, University Park, PA, USA
}

\begin{abstract}
Quorum-sensing (QS) interference is a novel therapy to fight bacterial infections that, unlike conventional antibiotic treatments, is focused on reducing the damage caused by pathogens (virulence) rather than focused on inhibiting their growth. Given this ideal, it was predicted that this approach will be impervious to or at least much less prone to resistance in bacterial populations. However, recently, resistance mechanisms against well-characterized quorum quenchers (QQs) have been found in the laboratory as well as in clinical strains, demonstrating that the rise of resistance against these kinds of compounds is possible. Nevertheless, it has been argued that even if resistance mechanisms against QS interference exist, this fact does not guarantee that resistance will spread. In the present work, we discuss recent insights derived from the latest experiments to address this question. In addition, we explain how environmental conditions like the stress produced by the host immune system may influence the selection of resistance and eventually lead to the selection of QS interference-resistant bacteria in a clinical setting.
\end{abstract}

The ISME Journal (2016) 10, 4-10; doi:10.1038/ismej.2015.84; published online 29 May 2015

\section{Resistance against quorum quenching with growth on private goods}

As quorum sensing (QS is used by bacterial pathogens to coordinate the expression of virulence factors at high cell densities, its inhibition (QS interference) was identified as a novel target to design antivirulence therapies, which aim to attenuate bacterial infections without decreasing growth, in contrast to antibiotics. One attractive feature of such therapies is that it was hypothesized that unlike growth inhibition-based therapies, anti-virulence therapies should be impervious to resistance (Rasmussen and Givskov, 2006; Bjarnsholt et al., 2010; Rasko and Sperandio, 2010). However, recently it was demonstrated that the generation and selection of resistance against quorum quenchers (QQs) occurs as readily as it does for antibiotics, under conditions in which growth is directly coupled to QS (García-Contreras et al., 2013b; Kalia et al., 2013; Maeda et al., 2012). Experimentally, these conditions are achieved by growing the bacterial pathogen Pseudomonas aeruginosa with adenosine as sole carbon source, as its

Correspondence: R García-Contreras, Department of Microbiology and Parasitology, Faculty of Medicine, Universidad Nacional Autónoma de Mexico, Mexico City 04510, Mexico.

E-mail: rgarc@bq.unam.mx

Received 10 February 2015; revised 29 March 2015; accepted 14 April 2015; published online 29 May 2015 catabolism is tightly dependent on a functional LasR QS system (Heurlier et al., 2005) and as it is metabolized internally by the QS-proficient bacteria. Therefore, adenosine is a private good that benefits exclusively those individuals that invest in producing the adenosine-catabolizing enzymes. As under these conditions, targeting QS is ultimately targeting growth, QQ is not different compared with antibiotic treatment and therefore prone to the development of resistance. This situation may occur far more than is generally recognized because, in general, QQ compounds may affect many phenotypes in addition to virulence. This conclusion is supported by the changes in gene expression produced by inhibitors such as brominated furanones (Hentzer et al., 2003; Ren et al., 2004), which inhibit multiple QS systems (Ren et al., 2001), anthranilic acid analogs (Lesic et al., 2007), which also decrease salt tolerance, and meta-bromo-thiolactone, which alters the expression of several tRNA and ribosomal genes (O'Loughlin et al., 2013).

Hence, QQ compounds developed thus far may affect growth in some manner and will probably lead to resistance, albeit at different rates depending on the pleiotropic affects.

Nevertheless, using combinations of $P$. aeruginosa QS-deficient mutants and QS-proficient wild-type strains to mimic the situation in which an ideal ( $100 \%$ effective and $100 \%$ specific) QQ is in use, Gerdt and Blackwell found that the QQ-resistant 
variant is selected when the population initially is equal parts of both kinds of cells; however, its selection is severely restricted when the resistant mimic is present at low cell proportions $(>1 \%)$. In these studies, the QS-deficient mutants mimic those cells sensitive to the quencher (sensitive or $S$ cells) because these cells have QS turned off, whereas the wild-type strain mimics those resistant individuals (resistant or R cells) able to produce QS signals and public goods in the presence of the quencher.(Gerdt and Blackwell, 2014) The method of using mimics originated with Mellbye and Schuster (2011). Like in precedent studies, adenosine-dependent growth of $P$. aeruginosa was studied, (Gerdt and Blackwell, 2014) and competition experiments in mixtures of QQ-sensitive (S) and QQ-resistant (R) variants were performed using different R/S ratios. Their results show that at very small initial R/S ratios, virtually no selection of the resistant phenotype was observed; this restriction of the resistance selection is explained by the limited production of homoserine lactone (HSL) signals by the QQR strains, which will be diluted in the population that consists primarily of QQS cells that do not produce HSL signals because of their sensitivity to the QQ compound. Signal dilution will prevent autoinduction and suppress the production of adenosine-catabolizing enzymes in the R strains.

However, a fundamental aspect of their experimental design (Gerdt and Blackwell, 2014) was culturing bacteria under conditions in which growth is extremely slow, probably due to at least in part by the poor oxygenation of cultures in 96 wells. Moreover, ideally, if QS had complete control over adenosine catabolism, then the S (QS-negative) cells should not grow at all in adenosine medium; hence, in any competition between $\mathrm{S}$ and $\mathrm{R}, \mathrm{R}$ should always dominate (Figure 1a). The fact that $S$ cells grow in adenosine suggests that QS and adenosine catabolism are not completely linked. Note the original work linking LasR with adenosine growth demonstrates that growth of the lasR mutant in adenosine is severely impaired (Heurlier et al., 2005).

The net result is that the relative fitness of the $R$ and $\mathrm{S}$ variants in the competition experiments was evaluated almost without allowing cells to replicate, as seen in Supplementary Figure 6 of their work, in which the QQS and QQR mimics were mixed in a 1:100 proportion. Critically, the ratio of both types of strains was determined after only about 1.5 total generations for the 1:100 proportion, and for the 1:1000 proportion, cell counts were carried out almost before any growth was achieved. Not surprisingly then, no changes were found with regard to the initial proportions, as those changes should only occur either by differential survival or death of the $\mathrm{S}$ and $\mathrm{R}$ strains. Certainly, to see changes in cell populations, active cell replication is required in these kinds of experiments as natural selection acts by favoring the offspring of the more fit variants in a population. In fact, similar experiments in adenosine medium performed by Mellbye and Schuster using the same $R$ and $S$ variants found that the fitness of the $R$ variant is greater than that of the $S$ variant and therefore, the enrichment of the R population (almost to $100 \%$ of cells) was achieved when the initial proportion of $\mathrm{R}$ was $1 \%$. However, unlike the study of Gerdt and Blackwell, the final proportions of $\mathrm{R}$ and $\mathrm{S}$ cells in the study by Mellbye and Schuster were determined after cultures reached the stationary phase; hence, their study allowed the bacteria to have at least three rounds of replication (Mellbye and Schuster, 2011). Note, although, another possibility that explains the growth of $S$ cells in adenosine is that mutations that help $S$ variants to grow in adenosine may arise, but this is not the intention of the experiments as the authors wish to compare $\mathrm{R}$ vs $S$ growth in the absence of further mutations. In addition, in our study using a QQ compound, furanone C-30, under conditions that promote growth on adenosine after mutations, the population of QQ-resistant mutants was enriched only after at least 12 cell-replication rounds (Maeda et al., 2012). Therefore, the results of Gerdt and Blackwell indicating that resistance to QQ compounds does not spread at low R cell numbers may be only valid under conditions where there is little growth.

Furthermore, by design, Gerdt and Blackwell (2014) created conditions where only one cell type can grow effectively, the R cells, with either public or private goods. When private goods are used for growth, R cells dominate. When growth is coupled to the utilization of public goods, although the presence of $S$ cells could slow $R$ cell replication by exploiting them, this exploitation depends on active production of public goods by R cells. When experiments start with very low percentages of $\mathrm{R}$ cells, these cells may also not grow effectively until they first synthesize the enzymes necessary for the degradation of amino acids and peptides and the QS-controlled exoproteases. Hence, in the poor growth conditions used, it is not clear that at the times evaluated $\mathrm{S}$ cells are exploiting $\mathrm{R}$ cells as $\mathrm{R}$ cells may not be actively producing the exoproteases yet.

Another aspect that may have a role in determining the availability of the QS signal produced by the QQ-resistant individuals is the population structure and bacterial distribution. In fact, in the same work, Gerdt and Blackwell (2014) evaluated the effect of population structure on the selection of resistance to QQ compounds under conditions in which growth was dependent on the production of extracellular proteases (public goods) and in which the QQ resistance was decoupled from signal production. In contrast, competition experiments using adenosine as a sole carbon source (a private good) (such as those in Gerdt and Blackwell, 2014; Mellbye and Schuster, 2011) were carried out in well-mixed conditions and under homogeneous environments in which the dilution of the signals would be the outcome. Nevertheless, in confined environments in 
a
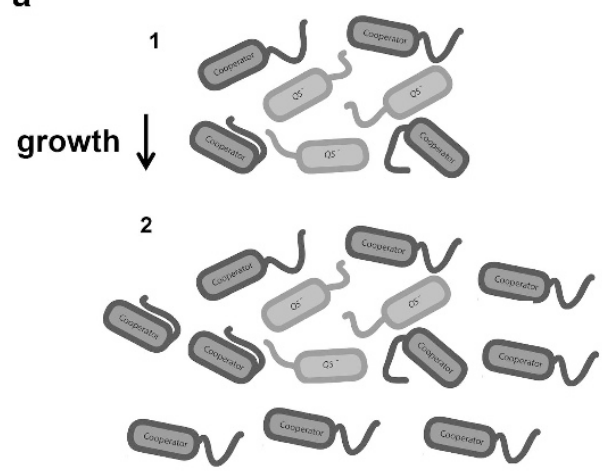

C

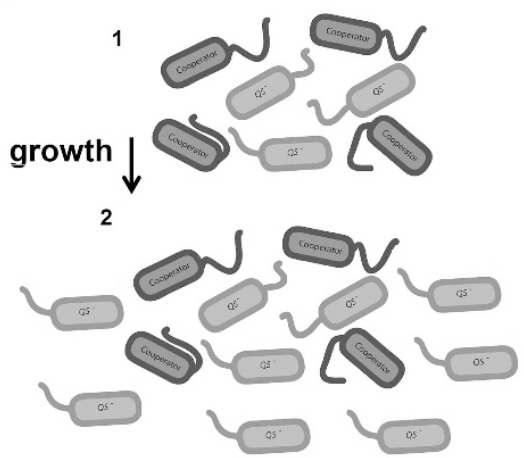

b

1

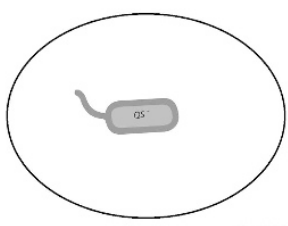

growth
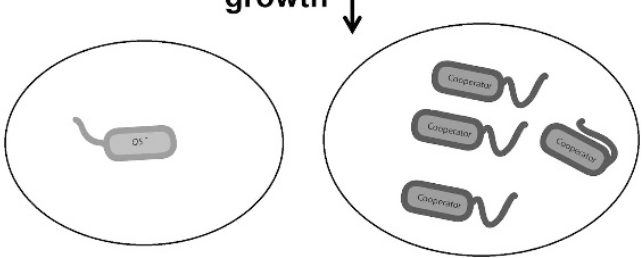

d

1
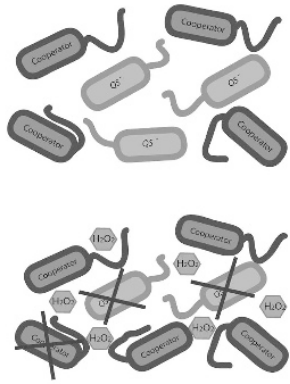

3

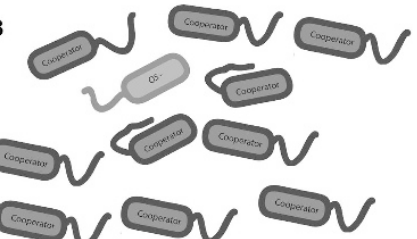

Figure 1 (a) Bacterial growth on a private good (for example, adenosine) with metabolism that is tightly dependent on QS in the presence of a QS inhibitor. Cooperator represents the variant that is resistant to the quorum-sensing inhibitor and able to quorum sense (R), whereas QS represents the variant sensitive to the QS inhibitor and unable to utilize QS (S). Sequential number of cells represents progressive times: (1) initial R and S population; (2) after growth, the R proportion is enriched as it is the only variant that can utilize the private good. (b) Bacterial grown on a private or a public good in confined environments in the presence of a QS inhibitor. Sequential number of cells represents progressive times: (1) initial R and S proportion isolates in different compartmentalized environments; (2) only R can grow, as S needs to exploit R for growing. (c) Bacterial growth on a public good (for example, bovine serum albumin) in the presence of a QS inhibitor. Sequential number of cells represents progressive times: (1) initial R and S population; (2) after growth, the S proportion is enriched as it exploits the public goods that is produced by the R cells. (d) Bacterial growth on a public good in the presence of stress (for example, oxidative stress from host cells) and in the presence of a QS inhibitor. Sequential numbers represent progressive times: (1) initial $\mathrm{R}$ and $\mathrm{S}$ proportion; (2) oxidative stress is applied to the bacterial population (note that the stress could be due other factors); (3) as $\mathrm{S}$ is much more sensitive to stress, $\mathrm{R}$ is selected.

which the diffusion of signals is not allowed, the production of a QS signal by a single cell is able to trigger its autoinduction (Carnes et al., 2010). Also, the spatial distribution of bacteria can be more important for sensing QS autoinducers than cell density (Hense et al., 2007). If in natural bacterial populations structured growth confines signal or public good diffusion, this could be relevant for the selection of QS-proficient strains and isolate them from potential social cheaters. (Figure 1b). Social cheaters are cells that do not contribute to the production of public goods but enjoy their benefit. By the same token, the isolation of cheaters may decrease their fitness if their growth is dependent on public goods produced by the cooperators.
In addition, non-water-soluble QS signals such as hydrophobic quinolones (for example, PQS), which are transported inside vesicles, perhaps diffuse less than more soluble molecules, such as lower molecular weightHSL, and PQS may be the main QS signal when $P$. aeruginosa is growing in sputum (which is viscous and hence also could limit diffusion) inside the lung of cystic fibrosis patients (Palmer et al., 2007). Furthermore, during infection, the spatial distribution of bacteria, especially in biofilms, could create microenvironments in which a few cells are partially isolated from the others and hence the diffusion of signals and public goods may be limited. Also, in P. aeruginosa biofilms, the QS signals PQS and HSL as well as some QS-controlled 
virulence factors are retained and concentrated in the biofilms by their binding with hydrophobic functional amyloid fibers (Seviour et al., 2015) Therefore, in biofilms, local semi-isolated bacterial subpopulations of QQR clones may accumulate sufficient signal levels to trigger the expression of their QS-dependent virulence factors and make them important for infections.

\section{Resistance against quorum quenching with growth on public goods}

In contrast to private goods, public goods are produced altruistically by cooperating individuals and can potentially benefit any member of the community regardless whether they invest or not in their production. Examples of public goods produced by pathogenic bacteria are siderophores, which deliver iron, and extracellular proteases, which cleave extracellular proteins into peptides and amino acids that can be internalized and metabolized. Several studies with $P$. aeruginosa have demonstrated that when growth is directly coupled to the production of public goods (for example, growth on protein as the sole carbon source), the appearance of selfish mutants (that is, social cheaters) is seen. These mutants are also found in clinical isolates from infections, indicating these processes could also exist under the relevant in vivo growth (Diggle et al., 2007; Sandoz et al., 2007). In addition, although the percentage of cheaters and cooperators in experiments often reach an equilibrium in which both kinds of cells coexist (Dandekar et al., 2013; García-Contreras et al., 2015; Sandoz et al., 2007), an overload of cheaters can collapse the population by overexploitation of the cooperators. This collapse in ecology is known as the tragedy of the commons.(Sandoz et al., 2007) Interestingly, in the context of $\mathrm{QQ}$, drugs that inhibit collectively beneficial traits (public goods), turn the susceptible population (S) into phenotypic cheats that will use the public goods generated by the resistant population that continues to utilize QS (Allen et al., 2014).

A recent experimental evolution work shows that extracellular quenching of the siderophore pyoverdine with gallium is more robust against the selection of resistance than equivalent antibiotic control. (Ross-Gillespie et al., 2014). However, some controls to further support that interesting conclusion were unfortunately not preformed. For example, (i) there were no experimental tests demonstrating that Ga remains outside the cells in both the in vitro and the in vivo experiments (with Galleria mellonella infections), which is important as $\mathrm{Ga}$ is readily internalized by $P$. aeruginosa (García-Contreras et al., 2013a; Kaneko et al., 2007); (ii) as at high concentrations $\mathrm{Ga}$ is bactericide, the highest in vitro Ga concentration used $(200 \mu \mathrm{M})$ may have killed all cells at the beginning of the experiment, therefore the next culture passes may have been inoculated mostly with dead cells; (iii) similarly, in vivo, the most effective doses of Ga used were extremely high

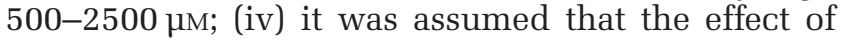
Ga was mainly on pyoverdine quenching but at least when $200 \mu \mathrm{M}$ Ga was used, no pyoverdine was detected; and (v) experiments in caterpillar hosts tested Ga but did not compare its effect with antibiotics.

Although to date there have been no experimental tests using QQs under conditions in which growth is dependent on the production of a public good to address whether resistance against these compounds may arise, it was hypothesized that under such conditions the spread of QQ resistance will be avoided or minimized (Allen et al., 2014). Moreover, in the works by Mellbye and Schuster (2011) and Gerdt and Blackwell (2014) that lacked a QQ compound, this situation was explored using the QQR and QQS mimics, grown in a medium that contained protein $(1 \%$ of bovine serum albumin (BSA)) as the sole carbon source; Mellbye and Schuster used BSA, and Gerdt and Blackwell used BSA plus casamino acids. Mellbye and Schuster found that the R population decreased when it was inoculated initially at 50 or $10 \%$ of the total population and that the proportion remained stable when it was inoculated at $1 \%$; hence, they demonstrated that if growth is dependent on the production of public goods, an ideal QQ therapy (under optimal laboratory conditions) will restrict the spread of resistance (Figure 1c). Unfortunately, at least for the initial 1\% $\mathrm{R}$ proportion, the $\mathrm{R}$ cells were inoculated at very low numbers and when such a low quantity of $\mathrm{R}$ cells are inoculated, their growth takes more time than the growth of S cultures without R (using a higher initial amount of $S$ inoculated cells). Their results suggest that in mixed cultures with very low initial $\mathrm{R}$ proportions, the growth of $\mathrm{S}$ cells could be independent of the growth of $\mathrm{R}$ cells. In addition, although no information was given about the number of bacterial generations developed under these conditions (Figure 1 of Mellbye and Schuster, 2011), the approximate final growth achieved in each experiment is available, so it can be estimated that there were around four generations of growth for every experiment; hence, the predicted outcome if cells are allowed to divide a higher number of times was not available. In addition, the presence of compensatory mutations that allow $S$ variants to grow faster in casein as a sole carbon source was not ruled out.

In the study by Gerdt and Blackwell, growth was dependent not only on protein as the sole carbon source but also on $0.1 \%$ of casamino acids, which would be a carbon source for both $\mathrm{R}$ and $\mathrm{S}$ variants of the population; hence, the presence of casamino acids decreases the selective pressure of producing extracellular proteases to generate suitable growth substrates (peptides and aminoacids). Moreover, when the $\mathrm{R}$ mimic was inoculated at 1:1000 and 1:10 000 proportions, after an initial fast growth of 
3-4 generations (probably by consuming casamino acids), no further growth was achieved after $200 \mathrm{~h}$ of culture; hence, the initial proportions remained stable.

Remarkably, Gerdt and Blackwell assessed the role of the spatial distribution in the selection of the $R$ phenotype by making bidimensional arrays of $\mathrm{R}$ and $S$ variants in agar plates and found that the $R$ variants are favored only if their interspersed monoclonal patches are large enough $(\sim 1 \mathrm{~cm}$ of diameter $)$ to ensure that the public good (elastase) remained available only to the $\mathrm{R}$ individuals, as smaller patches allowed the diffusion of elastase to reach the $S$ patches enhancing the $S$ growth at the expense of the R public good production. However, these experiments were carried out at $30^{\circ} \mathrm{C}$, which is lower than physiological temperatures, and perhaps the lower temperature decreased the specific rate of expression of the extracellular QS-controlled virulence factors such as exoproteases and also other compounds such as phenazines, membranedisrupting rhamnolipids (Grosso-Becerra et al., 2014) and toxic metabolites such as HCN (Castric, 1975), which may also influence the differential survival of the $\mathrm{S}$ and $\mathrm{R}$ variants as will be discussed next.

\section{Possible impact of environmental and host conditions on the selection of resistance against quorum quenching}

So far, only a few studies assessing the selection of QQ resistance in bacterial populations grown in either medium with a private or a public good have been made; however, under in vivo conditions, it may be possible that QS is simultaneously controlling the catabolism of metabolic private goods such as adenosine as well as the assimilation of extracellularly degraded public goods such as proteins. Interestingly, it was recently demonstrated that the social cheating phenomena observed in P. aeruginosa cultures grown on the public good BSA as the sole carbon source is suppressed by the addition of the private good adenosine, even at low concentrations of adenosine (Dandekar et al., 2013). Their results may then imply that in a QQ therapy of bacteria growing in a mixture of QS-controlled private and public goods as growth substrates (that is expected for most bacteria in vivo), the inhibiting effect of the quencher-susceptible population (S cells) over the resistant individuals (R cells) could be minimized and perhaps avoided (depending on the proportion of publically/privately used nutrients) and may therefore allow the spreading of resistance to QQ compounds.

Similar to the idea that the link between QS and private goods prevents cheating, recently, we demonstrated (García-Contreras et al., 2015) that the higher sensitivity of QS-deficient mutants to stress restricts the generation and spread of social cheaters (lasR mutants) in P. aeruginosa (Figure 1d).
For this work, the population of social cheaters is reduced due to their deficiency in the production of antioxidant enzymes; cells were grown on caseinate as a sole carbon source in the presence of oxidative stress. Therefore, linking QS and the stress response (which includes many environmental stresses for $P$. aeruginosa, not just oxidative stress), prevents social cheating.

Moreover, besides the metabolic restriction of cheaters proposed by Dandekar et al. (2013), asduring infections bacteria are subjected to high levels of stress produced by the immune system, it is likely that the stress may contribute both to the elimination of the cheaters and also allow QQR cells to spread under a QQ treatment (García-Contreras et al., 2015). For this work, we used the gold standard for inhibiting QS, the brominated furanone C-30. Indeed, we observed that QQR strains increase in number when both the QQ compound and stress are present. Specifically, in competitions between the mexR strain (a furanone C-30 QQR mutant, the $\mathrm{R}$ strain) and the C-30-sensitive strain (wild type, the $S$ strain), which were performed in LB medium (in which growth and survival are uncoupled to QS), we found that when C-30 was added in the absence of stress, the proportion of mexR mutant (R) cells increased only slightly after several culture passes. In contrast, when stress was applied (by adding $\mathrm{H}_{2} \mathrm{O}_{2}$ ) along with the QS inhibitor, the mexR (R) proportion increased dramatically from 25\% to almost 100\% (García-Contreras et al., 2015). Therefore, stress combined with QS inhibition results in strong selection for bacteria resistant to QS inhibitors as the QS response is intimately linked to the stress response.

In addition to the oxidative stress produced by the human host, cooperative individuals (under a QQ treatment, those bacteria that are resistant to the quencher molecules and capable of utilizing QS, so the R strain) also produce QS-controlled extracellular toxic compounds such as pyocyanin, which in turn, promote the generation of ROS and HCN which inhibit respiration. As the production of such virulence factors in $P$. aeruginosa is higher at $37^{\circ} \mathrm{C}$ than at lower temperatures and is high during infections, it is possible that the higher temperature allows the cooperative individuals ( $R$ strain) to decrease the number of non-cooperative cheaters during the infections, and therefore may be a mechanism that decreases the fitness of the QQ sensitive strains (S strain), as the QQR cells will produce the public good (for example, extracellular proteases) that will be available to the noncooperative cheaters by diffusion; however, also by diffusion, the toxic compounds will reach the cheaters and potentially decrease their growth. In fact, it was recently shown that in casein cultures, the production of HCN by cooperative wild-type $P$. aeruginosa individuals restricts the selection of social cheaters (lasR mutants) (Wang et al., 2015). Furthermore, recently it was discovered that in 
young biofilms, some $P$. putida cells produce HSL signals stochastically as self-regulatory signals, inducing the production of attached biosurfactants (private goods) that allow them to leave the biofilms by increasing their motility (Carcamo-Oyarce et al., 2015). Although this phenotype has not been observed yet among biofilms of bacterial pathogens, its existence would potentially allow, during QQ therapy, some of the QQR cells in biofilms to leave the biofilm, thereby, allowing them to 'escape' from QQS cells and avoid their opportunistic utilization of the public goods. This would promote the colonization of additional niches by QQR cells, which can potentially spread infections and resistance against QQ.

Other factors that could play a role in shaping natural bacterial populations in the environment (and perhaps also during infections) are biotic stressors such as bacteriophages. Recently it was described that Escherichia coli reduces the numbers of phage $\lambda$ receptors on the cell surface in response to N-acyl-lhomoserine lactone QS signals, leading to a significant increase in the frequency of uninfected survivor cells after a potent attack by $\lambda$ and other virulent phages. Hence, it would be possible that non cooperative individuals (that is, those cells that are sensitive to the QS inhibitor and thereby not using QS) will be more susceptible to phage attack than the cooperative ones (that is, those cells that are resistant to the QS inhibitor and thereby utilizing QS), providing yet another factor that may increase the population of $R$ variants over $S$ variants (Hoyland-Kroghsbo et al., 2013). However the effects of phages in R and S variants of pathogenic bacteria remain to be explored.

\section{Concluding remarks}

Although competition experiments with QSdeficient vs QS-proficient strain mimics has been useful to explore if the selection of QQ-resistant bacteria is possible, additional experiments are needed (Ruer et al., 2015), including the utilization of quenching molecules (Maeda et al., 2012). In this regard, it is argued that current QQs are not used as the available specific QQ (those with effects mainly on QS phenotypes without significant toxicity) often have modest QS inhibition activities whereas more potent inhibitors like C-30 have a higher toxicity (Gerdt and Blackwell, 2014). However, enzymes that disrupt QS signals (acylases and lactonases for HSL signals) are specific and potent (Chen et al., 2013); hence, they may be ideal to test different experimental conditions, ones that should be carefully designed and as close as possible to in vivo conditions. Nevertheless, a better approach could be the utilization of animal infection models to determine if QQ resistance can indeed arise and be selected under QQ treatments. Although there are some challenges in animal studies, like the determination of spatiotemporal bacterial population dynamics, such experiments would provide valuable information that will predict possible outcomes if eventually QQ therapies are implemented in the clinic. Alternatively, in vitro experiments using systems that mimic relevant tissues and organs such as the lungs and the GI tract, and so on from human-on-achip studies, may be useful. Also experiments with microfluidic devices will be very helpful, as they allow the examination of signaling among close and distal populations, and spatiotemporal arrays in bacterial populations can be created (Fernandes et al., 2010; Hong et al., 2012).

Significant potential problems to resistanceimpervious QQ therapies are the high variability observed in the expression of QS signals and genes (Defoirdt et al., 2010). Also, since successful QQ treatments in animal infections decrease pathogen bacterial loads (Defoirdt et al., 2010), this in principle imposes a strong selective pressure for the selection of resistance. Moreover, we have already found that some clinical strains are resistant against QQs, even without a pre-exposure to the QQ compounds (García-Contreras et al., 2013c; Maeda et al., 2012). Therefore, based on the above-discussed evidence, we think that perhaps QQ therapies may have lower resistance rates than antibiotics, but that designing an effective resistance-proof therapy to treat bacterial infections is far more challenging than it is currently anticipated. Ultimately, since evolution is a natural course of progression, we think that eventually any antibacterial/antivirulence therapy will select resistance, but perhaps we can find ways to guide such evolution or take advantage of it in order to create robust antibacterial/antivirulence strategies.

\section{Conflict of Interest}

The authors declare no conflict of interest.

\section{Acknowledgements}

This work was supported by grants from SEP/CONACyTMexico no.152794 to R-GC and by the Army Research Office (W911NF-14-1-0279) to TKW. TKW is the Biotechnology Endowed Chair at the Pennsylvania State University.

\section{References}

Allen RC, Popat R, Diggle SP, Brown SP. (2014). Targeting virulence: can we make evolution-proof drugs? Nat Rev Microbiol 12: 300-308.

Bjarnsholt T, Tolker-Nielsen T, Hoiby N, Givskov M. (2010). Interference of Pseudomonas aeruginosa signalling and biofilm formation for infection control. Expert Rev Mol Med 12: e11.

Carcamo-Oyarce G, Lumjiaktase P, Kummerli R, Eberl L. (2015). Quorum sensing triggers the stochastic escape 
of individual cells from Pseudomonas putida biofilms. Nat Commun 6: 5945.

Carnes EC, Lopez DM, Donegan NP, Cheung A, Gresham H, Timmins GS et al. (2010). Confinement-induced quorum sensing of individual Staphylococcus aureus bacteria. Nat Chem Biol 6: 41-45.

Castric PA. (1975). Hydrogen cyanide, a secondary metabolite of Pseudomonas aeruginosa. Can J Microbiol 21: 613-618.

Chen F, Gao Y, Chen X, Yu Z, Li X. (2013). Quorum quenching enzymes and their application in degrading signal molecules to block quorum sensing-dependent infection. Int J Mol Sci 14: 17477-17500.

Dandekar AA, Chugani S, Greenberg EP. (2013). Bacterial quorum sensing and metabolic incentives to cooperate. Science 338: 264-266.

Defoirdt T, Boon N, Bossier P. (2010). Can bacteria evolve resistance to quorum sensing disruption? PLoS Pathog 6: e1000989.

Diggle SP, Griffin AS, Campbell GS, West SA. (2007). Cooperation and conflict in quorum-sensing bacterial populations. Nature 450: 411-414.

Fernandes R, Luo X, Tsao CY, Payne GF, Ghodssi R, Rubloff GW et al. (2010). Biological nanofactories facilitate spatially selective capture and manipulation of quorum sensing bacteria in a bioMEMS device. Lab Chip 10: 1128-1134.

García-Contreras R, Lira-Silva E, Jasso-Chavez R, Hernandez-Gonzalez IL, Maeda T, Hashimoto T et al. (2013a). Isolation and characterization of gallium resistant Pseudomonas aeruginosa mutants. Int J Med Microbiol 303: 574-582.

García-Contreras R, Maeda T, Wood TK. (2013b). Resistance to quorum-quenching compounds. Appl Environ Microbiol 79: 6840-6846.

García-Contreras R, Martinez-Vazquez M, Velazquez Guadarrama N, Villegas Paneda AG, Hashimoto T, Maeda $\mathrm{T}$ et al. (2013c). Resistance to the quorum-quenching compounds brominated furanone C-30 and 5-fluorouracil in Pseudomonas aeruginosa clinical isolates. Pathog Dis 68: 8-11.

García-Contreras R, Nunez-López L, Jasso-Chávez R, Kwan BW, Belmont JA, Rangel-Vega A et al. (2015). Quorum sensing enhancement of the stress response promotes resistance to quorum quenching and prevents social cheating. ISME J 9: 115-125.

Gerdt JP, Blackwell HE. (2014). Competition studies confirm two major barriers that can preclude the spread of resistance to quorum-sensing inhibitors in bacteria. ACS Chem Biol 9: 2291-2299.

Grosso-Becerra MV, Croda-García G, Merino E, ServinGonzalez L, Mojica-Espinosa R, Soberón-Chávez G. (2014). Regulation of Pseudomonas aeruginosa virulence factors by two novel RNA thermometers. Proc Natl Acad Sci USA 111: 15562-15567.

Hense BA, Kuttler C, Muller J, Rothballer M, Hartmann A, Kreft JU. (2007). Does efficiency sensing unify diffusion and quorum sensing? Nat Rev Microbiol 5: 230-239.

Hentzer M, Wu H, Andersen JB, Riedel K, Rasmussen TB, Bagge $\mathrm{N}$ et al. (2003). Attenuation of Pseudomonas aeruginosa virulence by quorum sensing inhibitors. Embo J 22: 3803-3815.

Heurlier K, Denervaud V, Haenni M, Guy L, Krishnapillai V, Haas D. (2005). Quorum-sensing-negative (lasR) mutants of Pseudomonas aeruginosa avoid cell lysis and death. I Bacteriol 187: 4875-4883.
Hong SH, Hegde M, Kim J, Wang X, Jayaraman A, Wood TK. (2012). Synthetic quorum-sensing circuit to control consortial biofilm formation and dispersal in a microfluidic device. Nat Commun 3: 613.

Hoyland-Kroghsbo NM, Maerkedahl RB, Svenningsen SL. (2013). A quorum-sensing-induced bacteriophage defense mechanism. MBio 4: e00362-00312.

Kalia VC, Wood TK, Kumar P. (2013). Evolution of resistance to quorum-sensing inhibitors. Microb Ecol 68: 13-23.

Kaneko Y, Thoendel M, Olakanmi O, Britigan BE, Singh PK. (2007). The transition metal gallium disrupts Pseudomonas aeruginosa iron metabolism and has antimicrobial and antibiofilm activity. J Clin Invest 117: 877-888.

Lesic B, Lepine F, Deziel E, Zhang J, Zhang Q, Padfield K et al. (2007). Inhibitors of pathogen intercellular signals as selective anti-infective compounds. PLoS Pathog 3: 1229-1239.

Maeda T, García-Contreras R, Pu M, Sheng L, Garcia LR, Tomas $\mathrm{M}$ et al. (2012). Quorum quenching quandary: resistance to antivirulence compounds. ISME $J$ 6: 493-501.

Mellbye B, Schuster M. (2011). The sociomicrobiology of antivirulence drug resistance: a proof of concept. MBio 2.

O'Loughlin CT, Miller LC, Siryaporn A, Drescher K, Semmelhack MF, Bassler BL. (2013). A quorumsensing inhibitor blocks Pseudomonas aeruginosa virulence and biofilm formation. Proc Natl Acad Sci USA 110: 17981-17986.

Palmer KL, Aye LM, Whiteley M. (2007). Nutritional cues control Pseudomonas aeruginosa multicellular behavior in cystic fibrosis sputum. J Bacteriol 189: 8079-8087.

Rasko DA, Sperandio V. (2010). Anti-virulence strategies to combat bacteria-mediated disease. Nat Rev Drug Discov 9: 117-128.

Rasmussen TB, Givskov M. (2006). Quorum-sensing inhibitors as anti-pathogenic drugs. Int J Med Microbiol 296: 149-161.

Ren D, Sims JJ, Wood TK. (2001). Inhibition of biofilm formation and swarming of Escherichia coli by (5Z)-4bromo-5-(bromomethylene)-3-butyl-2-(5H)-furanone. Environ Microbiol 3: 731-736.

Ren D, Bedzyk LA, Ye RW, Thomas SM, Wood TK. (2004). Differential gene expression shows natural brominated furanones interfere with the autoinducer-2 bacterial signalling system of Escherichia coli. Biotech Bioeng 88: $630-642$.

Ross-Gillespie A, Weigert M, Brown SP, Kummerli R. (2014). Gallium-mediated siderophore quenching as an evolutionarily robust antibacterial treatment. Evol Med Public Health 2014: 18-29.

Ruer S, Pinotsis N, Steadman D, Waksman G, Remaut H. (2015). Virulence-targeted antibacterials: concept, promise, and susceptibility to resistance mechanisms. Chem Biol Drug Des; e-pub ahead of print 14 January 2015; doi:10.1111/cbdd.12517.

Sandoz KM, Mitzimberg SM, Schuster M. (2007). Social cheating in Pseudomonas aeruginosa quorum sensing. Proc Natl Acad Sci USA 104: 15876-15881.

Seviour T, Hansen SH, Yang L, Yau YH, Wang VB, Stenvang MR et al. (2015). Functional amyloids keep quorum-sensing molecules in check. J Biol Chem. 290: 6457-6469.

Wang M, Schaefer AL, Dandekar AA, Greenberg EP. (2015). Quorum sensing and policing of Pseudomonas aeruginosa social cheaters. Proc Natl Acad Sci USA 112: 2187-2191. 